\title{
A New Image Restoration Method for MUSER
}

\author{
Wei Wang $\mathbb{D}^{1,2}$ and Yihua Yan ${ }^{1,2,3}$ \\ ${ }^{1}$ National Astronomical Observatories, Chinese Academy of Sciences, China \\ ${ }^{2}$ Key Laboratory of Solar Activity of Chinese Academy of Sciences, China \\ ${ }^{3}$ University of Chinese Academy of Sciences, China
}

Correspondence should be addressed to Wei Wang; wwang@nao.cas.cn

Received 18 January 2019; Accepted 8 April 2019; Published 2 May 2019

Guest Editor: Junhui Fan

Copyright (C) 2019 Wei Wang and Yihua Yan. This is an open access article distributed under the Creative Commons Attribution License, which permits unrestricted use, distribution, and reproduction in any medium, provided the original work is properly cited.

\begin{abstract}
Solar radio images in decimeter wave range consist of many complicated components including a disk component, some bright and weak compact sources, and many diffuse features. Complicated structures combining these various components maybe cause restoration failure when using conventional algorithms. Furthermore, the images at different frequencies band are pretty different. Therefore, restoration method for solar radio image is different from other radio sources. Some image restoration methods were applied and obtained good results on Nancay radioheliograph images and Nobeyama radioheliograph images, and some new methods were introduced into processing these complicated solar radio images in recent years. For a new radioheliograph with ultrawide frequency band, new image restoration method which can maximize function of telescope is demanded. Different images could be obtained from the same visibilities data by using different weighting functions in imaging processing. In this paper, a new restoration method for solar radio image was proposed. Two images with different weighting functions from the same data are combined in this method. This restoration method has applied to data processing of Mingantu spectral radioheliograph.
\end{abstract}

\section{Introduction}

Mingantu spectral radioheliograph (MUSER) is a solar dedicated radio interferometric array that can produce high temporal resolution, high spectral resolution, and high spatial resolution radio images at decimeter to centimeter wave range [1]. Observation in routine has been carried out for 3 years since 2016; a mass of observational data and some image results have been obtained. The solar radio images are very different from other radio astronomical images. This is because there are a lot of different kinds of structures in solar radio images, such as a solar disk component; some bright compact sources like flares; some faint compact sources like radio emissions associated with $\mathrm{X}$-ray bright points and X-ray jets; many diffuse features like active regions, depressions associated with dark filaments, and plateaushaped enhancements in polar and coronal-hole regions [2]. Such complicated structures could cause restoration failure and increased computational time when using conventional algorithms. Using standard CLEAN algorithm and its evolutional algorithm cannot obtain good results for MUSER images with ultrawide frequency band.

Some methods developed for other radioheliographs in the world give us some experiences to solve this problem. A new improved multiscale CLEAN algorithm was applied to Nancay radioheliograph $(\mathrm{NRH})$ data. This algorithm restores solar image with a wide spectrum of spatial scales by using 5 or 6 uv-filters including a central disk and 4 or 5 rings, and it is less time-consuming than the ordinary CLEAN procedure [3]. About Nobeyama radioheliograph (NoRH), there are 3 methods to process image; the first of the three used standard CLEAN algorithms, optimized to flares using dirty models, which mean the dirty images of model Gaussian sources with various sizes [4]. The Steer algorithm was the second method, which is better for diffuse radio sources [2]. The third method is to attain the expected resolution in partial images and introduces two-step CLEANs [5].

According to our knowledge of solar physics, we know that solar radio image at different frequency band corresponds to positions in different height from solar surface. 
That means solar radio images at low frequency band are much different than ones at higher frequency band. The frequency of MUSER is $0.4 \mathrm{GHz}$ to $15 \mathrm{GHz}$, which is much wider than NRH and NoRH. It is hard to process these data at wide frequency band with the same method. Therefore, methods for NoRH and NRH could not be applied directly to process MUSER data. Therefore, a new image restoration method is proposed to process MUSER data in wide frequency band in this paper.

\section{Restoration Method}

Usually the weighting function is specified in many Fourier transform imaging programs to fine tune the beam shape [6]. The beam shape can be controlled or be extended by the weighting function. The beam shape means spatial resolution. The image with different spatial resolution can be processed from the same visibilities by using different weighting functions. The main idea of this restoration method is combination of two images which applied different weighting functions and different clean procedures.

2.1. The Weighting Functions. The points in $\mathrm{u}-\mathrm{v}$ coverage are always insufficient for an interferometric array, especially for solar observation in snapshot mode. The radio image of the sun is changing with time, it is impossible to combine the visibilities with longer integration time to improve quality of image. The integration time for solar observation is only a couple of milliseconds. The distribution of points in $\mathrm{u}-\mathrm{v}$ plane is not uniform. The density weighting function can be used to compensate for the clumping of data in $\mathrm{u}-\mathrm{v}$ plane by weighting by the reciprocal of the local density [6]. There are two choices for the weighting function in a general way, natural weighting and uniform weighting.

For natural weighting, all of points in $\mathrm{u}-\mathrm{v}$ plane were treated equally. For solar observation, a large member of short baselines are necessary for achieving the solar disk and largescale structure well. The number of MUSER antennas within 100 meters is about 40 percent. Therefore, natural weighting emphasizes the data from the short baselines. The shape of beam intends to be extended, and a relative wide beam size will occur in the image with natural weighting. But the advantage of extended beam size is to get much better signalto-noise ratio for detecting the weak sources, such as dark filaments and coronal hole.

For uniform weighting, points within denser areas in $\mathrm{u}-\mathrm{v}$ plane have lower weights than others. The density distribution of central area is dense and the surrounding is sparse gradually. That means the weight of data from short baseline is lower than from long baseline. It makes against observing large-scale structure of solar image. Compared to natural weighting, the beam size with uniform weighting is less than with natural weighting and is better for obtaining high spatial resolution for the compact sources in the sun, such as flare and X-ray jets.

Sometimes, these two weighting functions are insufficient especially in the snapshot mode of observing. For super uniform weighting, robust weighting was developed to be response to these problems [6]. In this image restoration method, only natural weighting and uniform weighting have been taken into account.

2.2. The Deconvolution. The visibilities output from correlator of an interferometry can be used to produce an image of distribution of sky brightness. But the quality of image is insufficient due to the loss of visibilities caused by limited number of antennas. So, the deconvolution is necessary for radio images observed by interferometric array. The CLEAN algorithm and some derivative algorithm are the predominant algorithm used for deconvolution of radio images. In this restoration method, standard CLEAN algorithm and Steer algorithm are combined for MUSER image.

The CLEAN algorithm was devised by J. Hogbom in 1974 [7]; it intends to create a model of the true "skybrightness distribution" from the "dirty image" which is the observed image using the "dirty beam". The model is called the "clean image" and is created using the "clean beam," which represents an ideal PSF. It is typically a Gaussian function of the same FWHM as the central component of the dirty beam [8]. The CLEAN algorithm can get better results for point sources or point-like sources. But the extended sources sometimes could not be recovered because of lacking the extremely short or zero spacings. For solar observation, solar disk component always existed in images. So standard CLEAN procedure is insufficient to process the data of MUSER.

The Steer algorithm is proposed by Steer et al. in 1984 [9]; it was applied to the data processing in daily solar images in the automatic data processing routine of the Nobeyama radioheliograph [2]. The Steer algorithm deals with radio sources in a group rather than individually. Components brighter than a certain fraction of the peak brightness in the dirty image are regarded as a group, called a contour-trim; the contour-trim is convolved with the dirty beam to create a dirty contour-trim, which is extracted from the dirty image [9]. The Steer algorithm needs less computational time than standard CLEAN algorithm due to group subtraction other than point subtraction.

2.3. Combination of Weighting and Deconvolution. According to analysis of different weighting functions and deconvolution algorithm, uniform weighting and standard CLEAN algorithm intended to process point sources and point-like sources, and natural weighting and Steer algorithm intended to deal with extended sources. There are various structures including a disk, compact sources, and diffuse sources in solar radio images as mentioned in the Introduction of this paper. Therefore, the main idea is combination of different weighting functions and deconvolution algorithms; a new synthetic restoration method is proposed and proceeds as follows.

(1) Remove partial solar disk component

(2) Obtain the first dirty image from the data with uniform weighting 

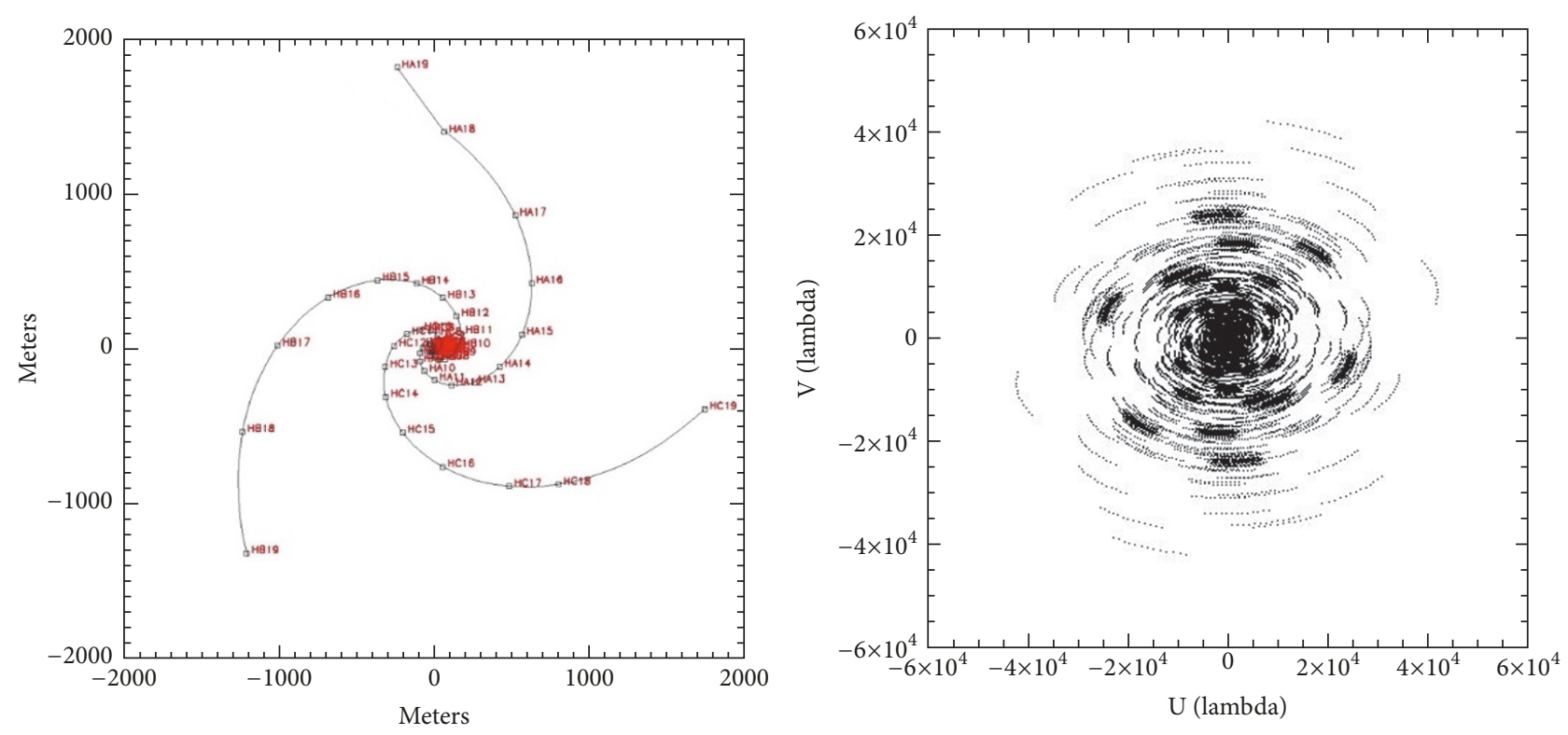

FIGURE 1: (Left) positions of antennas and (right) u-v coverage.

(3) A Hogbom CLEAN algorithm is performed on the first dirty image; get a clean image

(4) Obtain the second dirty image from the data with natural weighting

(5) A Steer algorithm is performed on the second dirty image; get another clean image

(6) Add the two clean images together; add the residuals of these dirty images to the clean image

(7) Add back the solar disk component and get the final image

A solar disk component created by convolution of a model of solar disk and the dirty beam was subtracted from the visibilities data before applying this method and added back in clean images finally. It must be noted that the component subtracted is not all solar disk and is just part of solar disk. The reason of this is more easily detecting the weak sources in the solar disk than the solar disk removed completely. Therefore, some bright and faint compact sources, many diffuse structures, and partial disk were left for restoration. Besides that the peak values of the two clean images should be scaled to the same before adding together.

\section{Result of MUSER Observation}

This new restoration method was performed on observational data of MUSER. The data on July 5, 2016, was processed with this restoration method. The frequency of this observation is $4.2 \mathrm{GHz}$. The sun is very quiet without any bursts from flux density observed by Nobeyama radio polarimeters and radio spectrum of MUSER. To improve quality of image, the 2-hour data with 10-minute cadence were merged together. The integration time of each point in $\mathrm{u}-\mathrm{v}$ plane is 60 milliseconds. The antenna configuration and $\mathrm{u}-\mathrm{v}$ coverage of this data are shown in Figure 1. The dirty image with natural weighting function and clean image using Steer algorithm are shown in Figure 2. The dirty image with uniform weighting function and clean image using Hogbom CLEAN algorithm are shown in Figure 3. The final image using this restoration method and image observed with NoRH are shown in Figure 4.

From the final image using this restoration method applied to MUSER observational data, the quality of this image is better than only using one deconvolution algorithm, similar result to NoRH. There are differences between images of MUSER and NoRH due to different observational frequency.

\section{Discussion}

Only natural weighting and uniform weighting are performed in this restoration method in this paper. Solar radio images at different frequency are different. Also, the solar image in a different phase of the solar activity cycle, such as solar minimum and maximum, is much different. Therefore, some other weighting functions, such as robust weighting, perhaps need be considered on the data with other frequency bands in the future. In addition, the cleaned image by different weighting function is added together with equal weighting. Adjusting different weights for different cleaned images makes this restoration method more general for processing different solar images.

\section{Conclusion}

MUSER is a solar dedicated interferometry which is called new generation solar radio telescope. It covered decimeter to centimeter wave range. Some new restoration methods or algorithms are needed to meet the requirement of the 

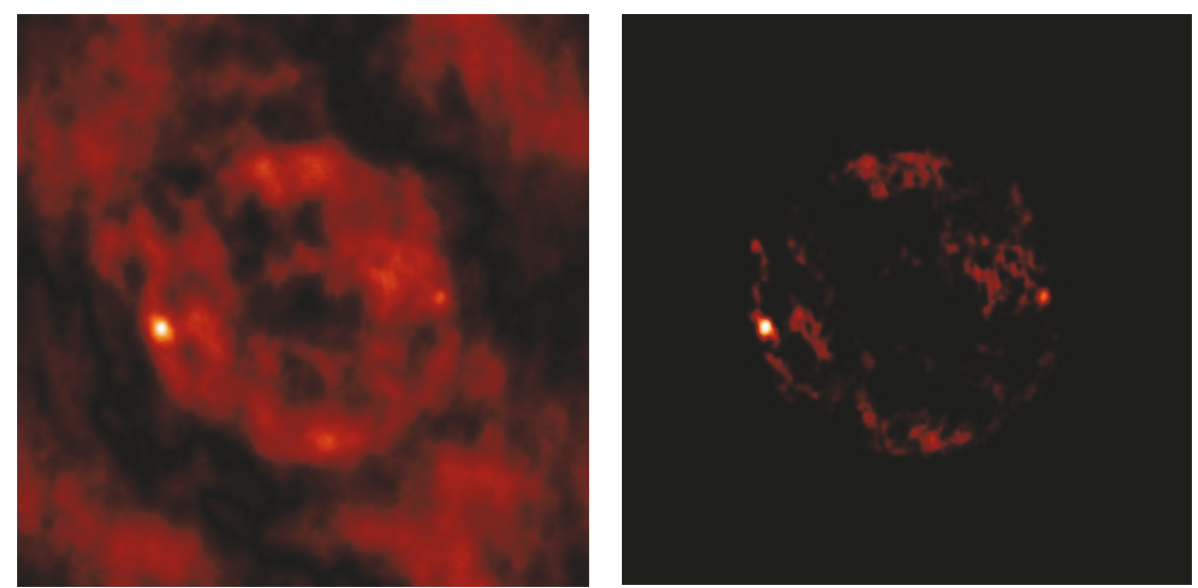

FIGURE 2: (Left) dirty image with natural weighting and (right) clean image using Steer algorithm.
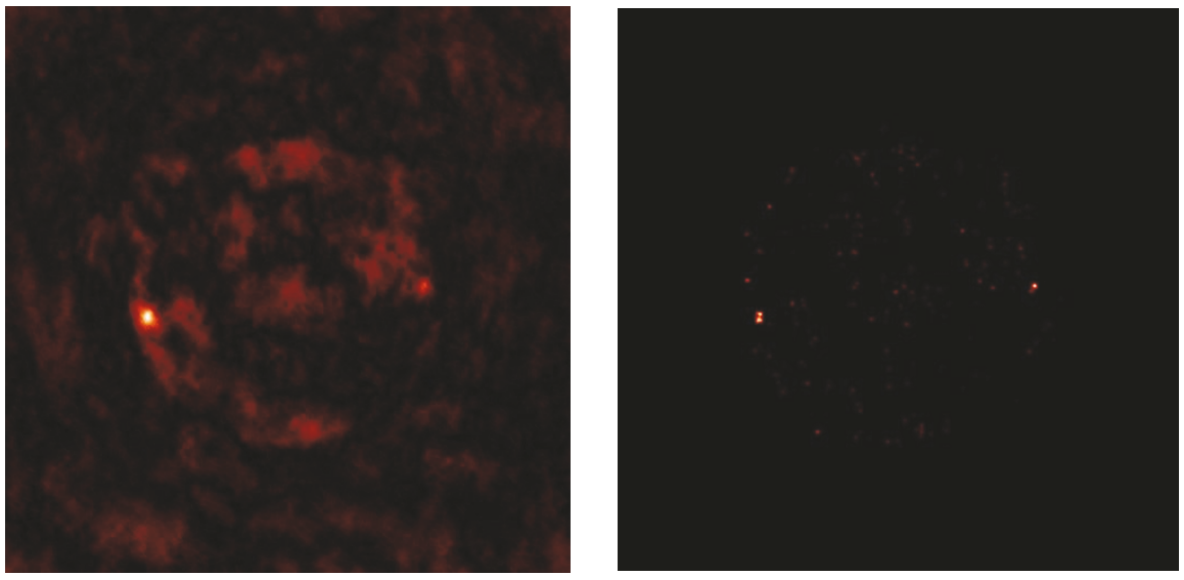

FIGURE 3: (Left) dirty image with uniform weighting and (right) clean image using Hogbom CLEAN.
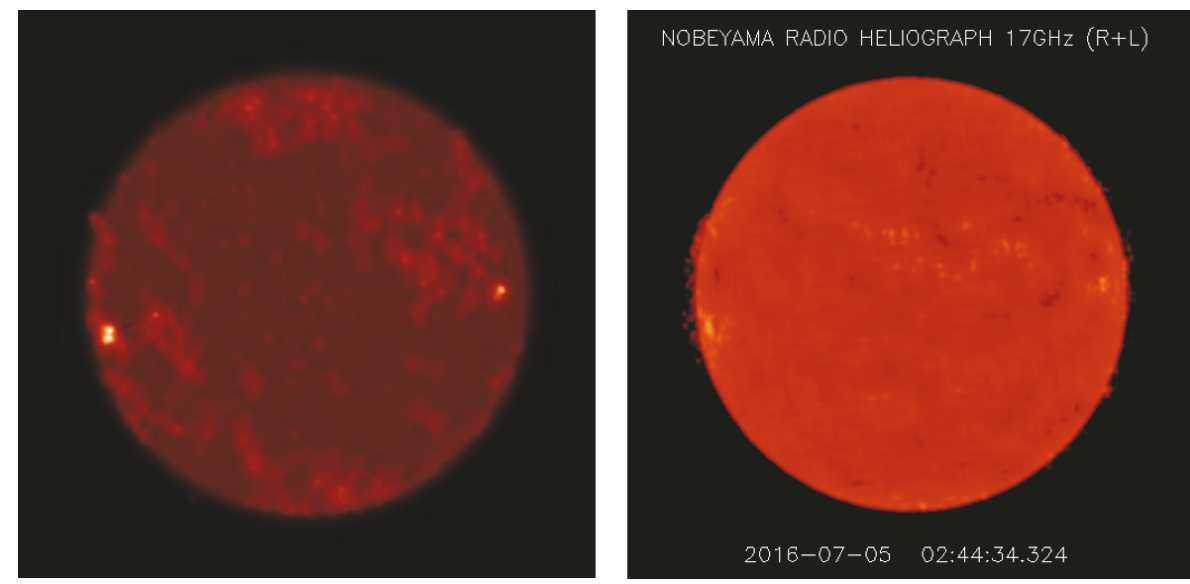

FIgURE 4: (Left) the final image of MUSER and (right) image of Nobeyama radioheliograph. 
ultrawide frequency band and complicated structures of solar image. In this paper, a new restoration method is proposed and applied to the data of MUSER, obtaining some results.

\section{Data Availability}

The data of MUSER and processing manuscripts are open access for everyone concerned.

\section{Conflicts of Interest}

The authors declare that they have no conflicts of interest.

\section{Acknowledgments}

Prof. Dale Gary gratefully acknowledges comments and suggestions to improve the restoration method. This work is supported by National Natural Science Foundation of China, grants 11433006, 11790301, 11790305, and 11773043, and also supported by open research project of NAOC-Ali Astronomical Big Data Joint Research Center.

\section{References}

[1] W. Wang, Y. Yan, D. Liu et al., "Calibration and data processing for a chinese spectral radioheliograph in the decimeter wave range," Publications of the Astronomical Society of Japan, vol. 65, S18, 2013.

[2] H. Koshiishi, "Restoration of solar images by the Steer algorithm," Astronomy \& Astrophysics, vol. 412, no. 3, pp. 893-896, 2003.

[3] C. Mercier, "High dynamic solar radio images by combining visibilities from the Giant Meterwave Radio Telescope (GMRT) and the Nancay Radioheliograph (NRH)," in Proceedings of the Annual meeting of the French Society of Astronomy and Astrophysics, vol. P75, 2006.

[4] Y. Hanaoka, "Processing of the Nobeyama Radioheliograph Data," in Proceedings of Kofu Symposium, pp. 35-43, Kofu, Japan, 1993.

[5] K. Fujiki, Ph.D. Dissertation, the Graduate University for Advanced Studies, 1998.

[6] R. Perley, F. Schwab, and A. Bridle, "Synthesis imaging in radio astronomy," Astronomical Society of Pacific Conferences Series, vol. Volume 6, 1999.

[7] J. A. Hogbom, "Aperture synthesis with a non-regular distribution of interferometer baselines," Astronomy \& Astrophysics, vol. 15, p. 417, 1974.

[8] U. J. Schwarz, "Mathematical-statistical Description of the Iterative Beam Removing Technique (Method CLEAN)," Astronomy \& Astrophysics, vol. 65, p. 345, 1978.

[9] D. G. Steer, P. E. Dewdney, and M. R. Ito, "Enhancements to the deconvolution algorithm 'CLEAN"' Astronomy \& Astrophysics, vol. 137, no. 2, pp. 159-165, 1984. 

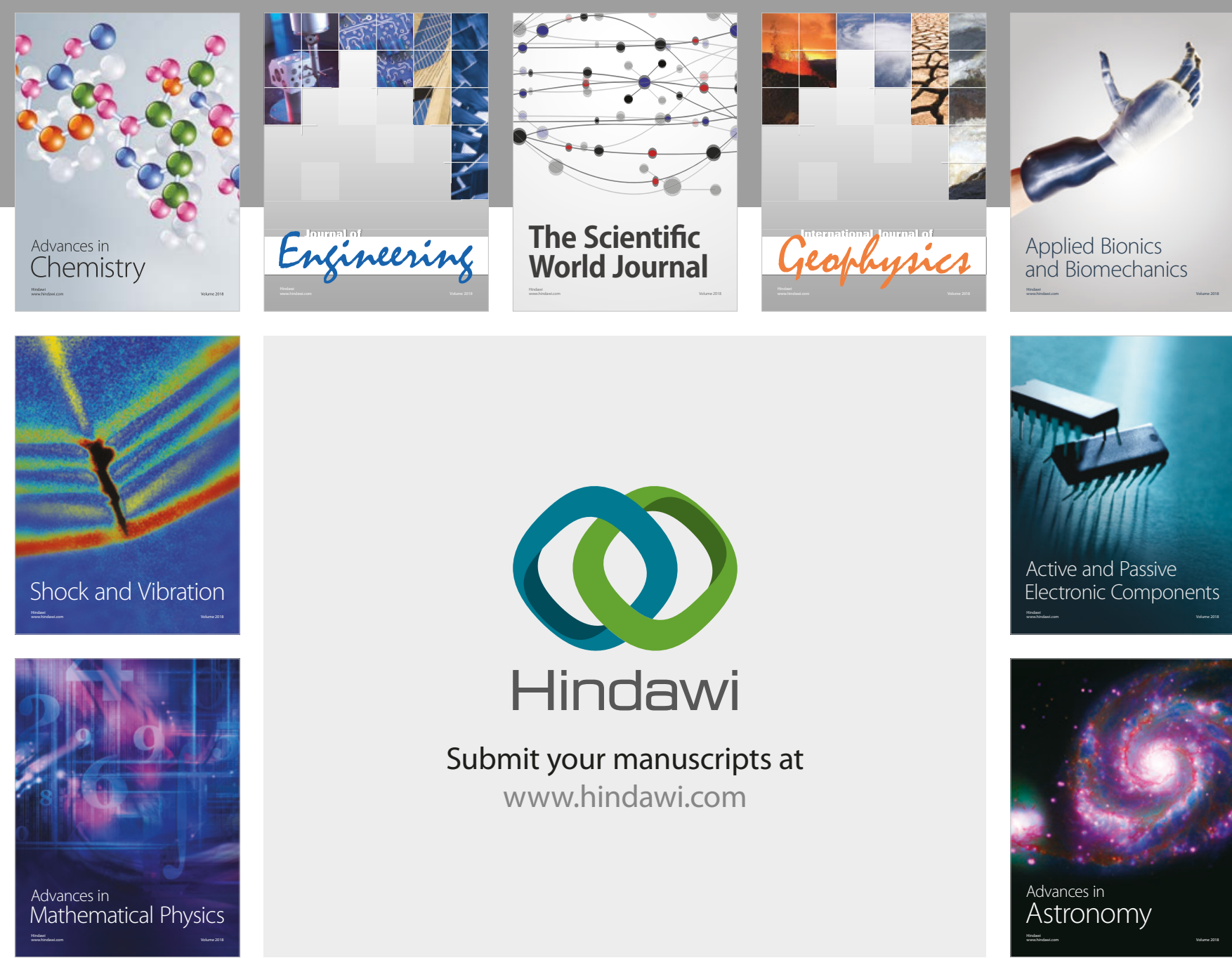

Submit your manuscripts at

www.hindawi.com

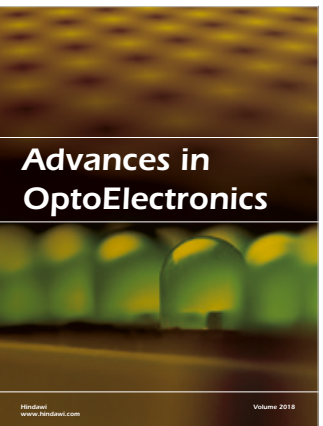

\section{Rotcting Machinery}
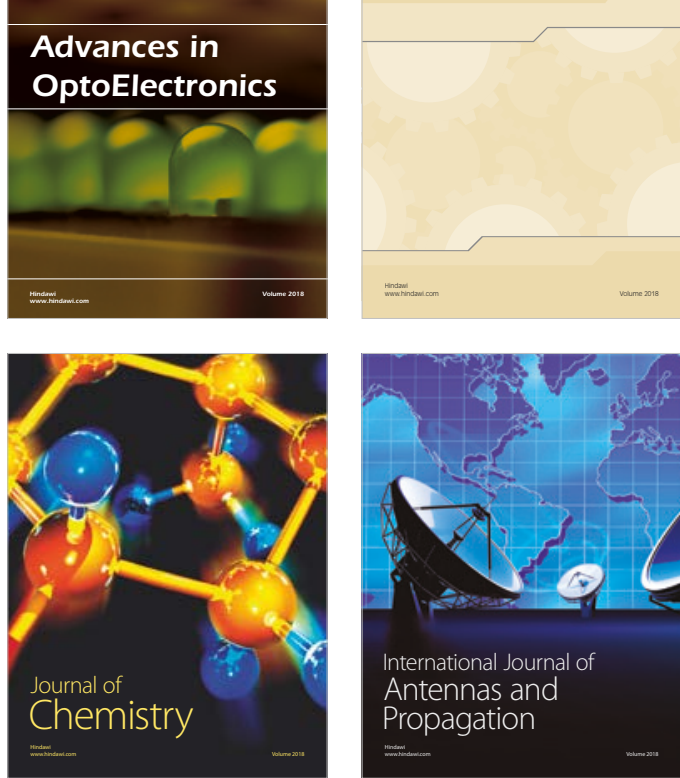

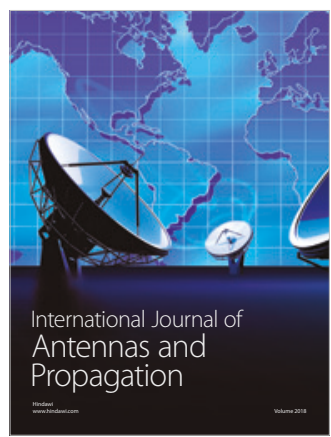

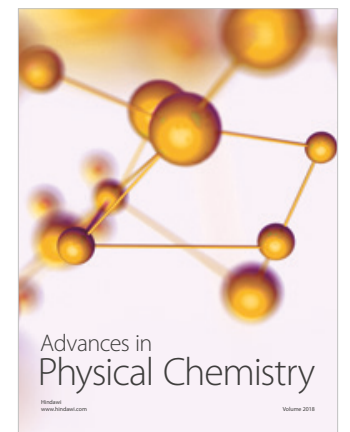

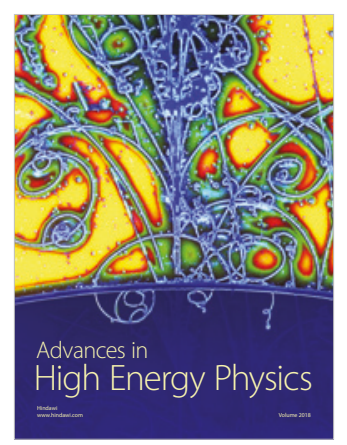

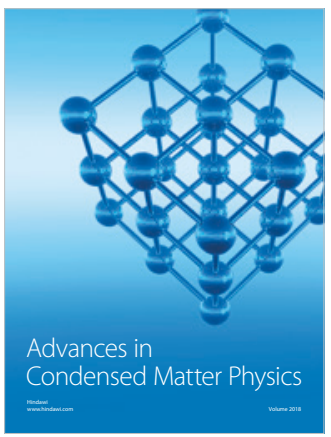

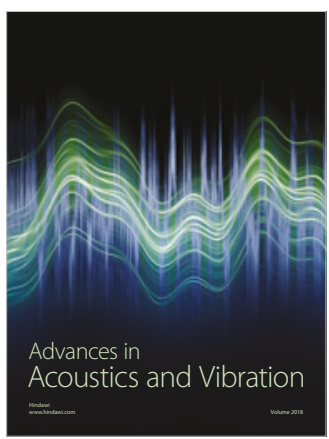

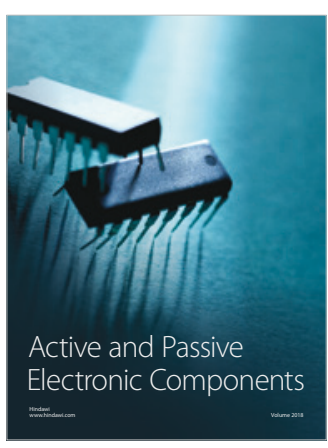
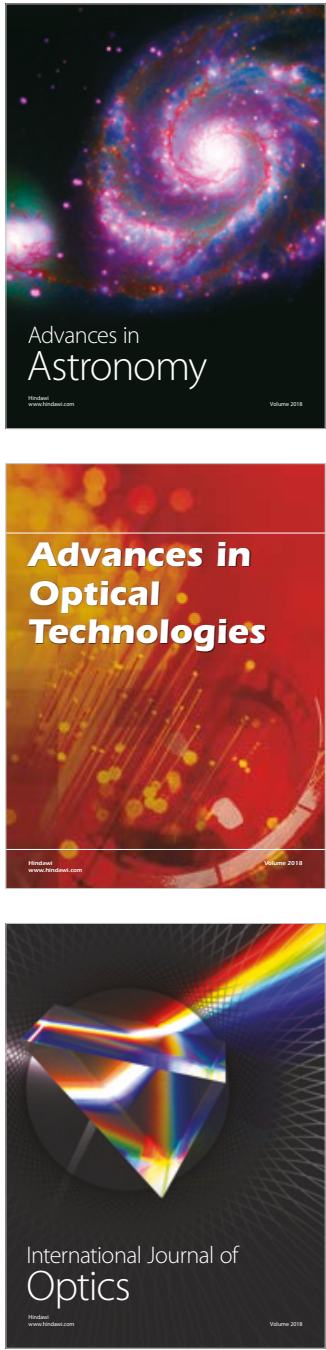